\title{
Iridocorneal endothelial syndrome: Cogan -Reese syndrome. A case report
}

\author{
Kumar $\mathbf{K}^{1}$, Ingle $\mathbf{R}^{2}$ \\ ${ }^{1}$ Dr Kavita Kumar, Associate professor, Department of Ophthalmology, ${ }^{2}$ Dr Rashmi Ingale, PG Resident. Both affiliated \\ with Regional Institute of Ophthalmology Gandhi Medical College, Bhopal, MP, India
}

Address for Correspondence: Dr Kavita Kumar, Email: kavita.kumar43@gmail.com

\begin{abstract}
ICE syndrome is a rare progressive unilateral disease occurring in young middle aged females. Corneal edema with uveal involvement is key feature. Secondary glaucoma is main sight threatening complication. Management depends on severity of ocular findings and is targeted to control corneal edema and secondary glaucoma medically and surgically.
\end{abstract}

\section{Introduction}

ICE (iridocorneal endothelial) syndrome is congenital condition, usually unilateral, seen in middle aged females, not associated with systemic disease ${ }^{1}$. Patient complains of blurred vision ocular pain.

Signs include varying amount of corneal oedema, iris heterochromia and distorted pupil ${ }^{1}$. ICE syndrome consists of 3 similar syndromes: Cogan Reese Syndrome-Iris Nevus, Chandlers syndrome -mild iris thinning, greater corneal edema, Essential Iris atrophyprogressive thinning, holes, corectopia ${ }^{1}$.

\section{Case report}

We report an 18 year old patient presented to our clinic with blurred vision and pain persisting for 1 month in the left eye.

Patient was apparently all right 1 month back when she had episode of pain redness followed by progressive diminution of vision and whitening of cornea. Family and personal history were not contributory.

No history of trauma or any major systemic illness like tuberculosis, leprosy was available. Stigmata of neurofibromas /Stills disease / other congenital anomalies were absent.

Manuscript received: $23^{\text {rd }}$ Aug 2013

Reviewed: $16^{\text {th }}$ Sept 2013

Author Corrected: $19^{\text {th }}$ Oct 2013

Accepted for Publication: $10^{\text {th }}$ Nov 2013
Examination-

Vision in right eye was normal. Slit lamp examination of right eye was normal. Left eyeball was prominent (exophthalmos) and exophoria with moderate ptosis. Vision was decreased to perception of light with defective projection in superior and nasal quadrant.

Left eye had following additional findings:

1. Unilateral Severe Corneal edema.

2. Iris Nevus was present on the anterior surface of iris, from 12 to 4 O'clock position extending up to pupillary margin.

3. Ectropion uveae.matted appearance of iris stroma, loss of iris crypts in temporal quadrant.

4. Left eye unilateral secondary glaucoma ( IOP $42 \mathrm{~mm}$ of Hg schiotz).

On investigation by Fundus and gonioscopy Right Eye was normal and could not be performed on LE due to severe corneal odema.

B scan of RE was normal and LE showed Macrophthalmia with axial length $25 \mathrm{~mm}$. Other investigations like X-ray skull, bilateral knee, USG abdomen, paediatric, cardiology and orthopaedic examinations were within normal limits.

All these findings were in favor of Iris nevus (CoganReese) syndrome. 


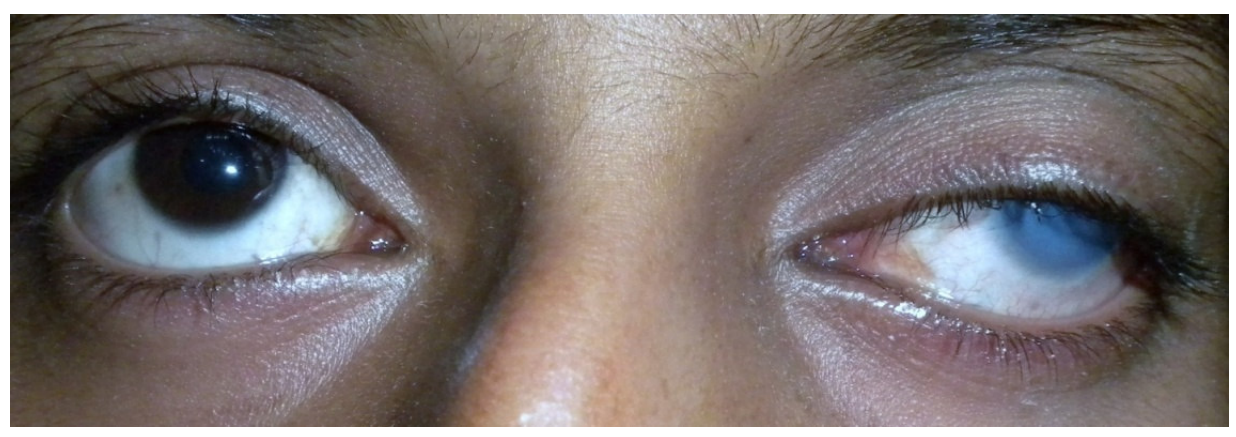

Fig1: Left eye showing exophthalmus, exophoria \& ptosis

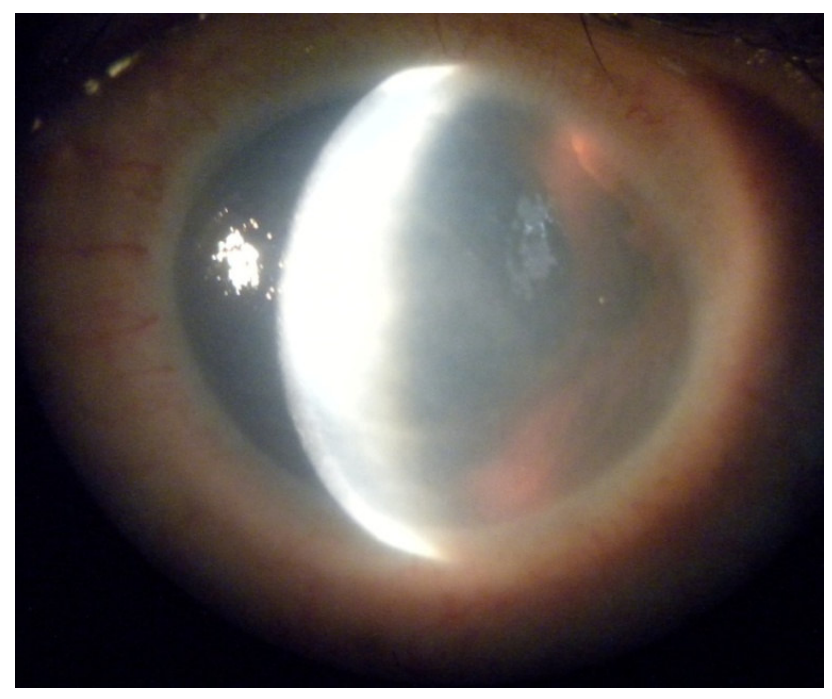

Fig 2: Severe corneal edema with Iris nevus on anterior surface of eyes from 12 to 4 O'clock position.

In our patient medical management was done with local and systemic anti glaucoma drugs and intraocular pressure came down to $14.6 \mathrm{~mm}$ of $\mathrm{Hg}$ in left eye after 11 days with symptomatic relief. Surgical intervention for glaucoma and corneal edema was not considered owing to poor visual prognosis.

Our patient is being followed up for secondary glaucoma.

\section{Discussion}

Iridocorneal endothelial syndrome is congenital condition, usually unilateral, seen in middle aged females, not associated with systemic disease. Patient complains of blurred vision, ocular pain, corneal edema, secondary glaucoma, heterochromia and distorted pupil ${ }^{1}$ ICE syndrome consists of 3 similar syndromes ${ }^{2}$.

\section{Cogan Reese Syndrome- Iris Nevus}

2.Chandlers syndrome -mild iris thinning, greater corneal edema.

3. Essential Iris atrophy-progressive thinning, holes, corectopia.

Etiology is unknown. Epstein Barr virus and Herpes simplex viruses have been found serologically in patients of ICE syndrome ${ }^{3}$. These viruses are suggested to play a role in cell necrosis and in transformation of endothelial cells. In ICE syndrome the corneal endothelium undergoes an epithelioid metaplasia that migrates in a membrane form ${ }^{2}$. If it migrates over the anterior chamber angle, contraction of this membrane pulls the iris toward the cornea causing a synechial closure of the angle leading to secondary glaucoma ${ }^{4}$. When the abnormal corneal endothelium spreads on to the iris surface, the contraction causes atrophy and corectopia. Pigmented iris nodules are also produced by this contracting abnormal endothelial membrane. Corneal edema occurs due to the subnormal endothelial pump function.

Cogan -Reese syndrome named after David Glendenning Cogan and Aligernon Reese, is an extremely rare eye 
disorder seen in young and middle aged females, usually affecting one eye, inciduous in onset, characterized by a matted or smudged appearance of the surface of iris, development of modular iris nevi, ectropion uveae, peripheral anterior synechiae, and/or glaucoma. Secondary glaucoma may lead to vision loss.

This disorder most frequently appears in young and middle-agedfemales, usually affecting one eye (unilateral) and developing slowly over time ${ }^{1}$. The differential diagnosis of multiple pigmented iris nodules include Neurofibromatosis, melanomas, inflammatory nodules of leprosy, syphlis, tuberculosis and sarcoidosis 5

Chandlers syndrome - This variant shows minimum or no iris atrophy, mild corectopia may be present. The cornel edema may be more pronounced, giving pleated silver appearance of posterior corneal surface.

Essential Iris Atrophy-This variant is characterized by severe iris atrophy resulting in heterochromia, marked corectopia, ectropion uveae, psuedopolycoria \& iris hole formation are hallmark of essential iris atrophy ${ }^{3}$

Treatment- Main concern of management is secondary glaucoma which occurs in about $50 \%$ of ICE syndrome patients and is major cause of visual $\operatorname{loss}^{6}$. Management options are specific to each case and should be tailored to degree and severity of secondary glaucoma and corneal edema.

\section{Conclusion}

ICE is a rare, progressive, congenital, unilateral disease, of unknown etiology occurring in middle aged females.
Main clinical feature is ocular pain, blurred vision. Secondary glaucoma is a sight threatening complication. Management is targeted to reduce corneal edema, and control secondary glaucoma medically. In severe cases surgical correction of glaucoma and penetrating keratoplasty is required to achieve and maintain good vision.

\section{References}

1. Laganowski HC, Kerr Muir MG \& Hitchings RA (1992): Glaucoma and the Iridocorneal 237 endothelial syndrome. Arch Ophthalmol 110: 346-350.

2. Wilson MC, Shields MB.A comparison of the clinical variations of the iridocorneal endothelial syndrome.Arch Ophthalmology 1989; 107(10): 1465-8

3. Campbell DG, Shields MB.The corneal endothelium and spectrum of essential iris atrophy.Am J ophthlmol.1978;86:317-24

4. Denis, P. Iridocorneal endothelial syndrome and glaucoma J Fr Ophthalmol.2007 Feb; 30 (2):189-95

5. Yıldız O” zdemir, Feyza O” nder, C. Banu Cos,ar, Alp Usubu"tün and Gücan Kural. Clinical and histopathologic findings of iris nevus (Cogan-Reese) syndrome - ActaOphthalmol. Scand. 1999: 77: 234-237

6. Yanoff M. In discussion of Shields MB, McCracken JS, Klintworth GK, Campbell DG. Corneal edema in essential iris atrophy. Ophthalmology. 1979;86::1549-55

\section{How to cite this article?}

Kumar K, Ingle R Iridocorneal endothelial syndrome: Cogan -Reese syndrome. A case report. Int J Med Res Rev 2013;1(5):261-263. doi:10.17511/ijmrr.2013.i5.08. 